\title{
Expanding the role of research evidence in family planning policy, program, and practice decisionmaking
}

\author{
Karen Hardee \\ Population Council \\ Kelsey Wright \\ Population Council
}

Follow this and additional works at: https://knowledgecommons.popcouncil.org/departments_sbsr-rh

Part of the Demography, Population, and Ecology Commons, Family, Life Course, and Society Commons, and the International Public Health Commons How does access to this work benefit you? Let us know!

\section{Recommended Citation}

Hardee, Karen and Kelsey Wright. 2015. "Expanding the role of research evidence in family planning policy, program, and practice decisionmaking," Working paper. Washington, DC: Population Council, The Evidence Project. 
Expanding the Role of Research Evidence in Family Planning Policy, Program, and Practice Decision-making

Karen Hardee Kelsey Wright

September 2015

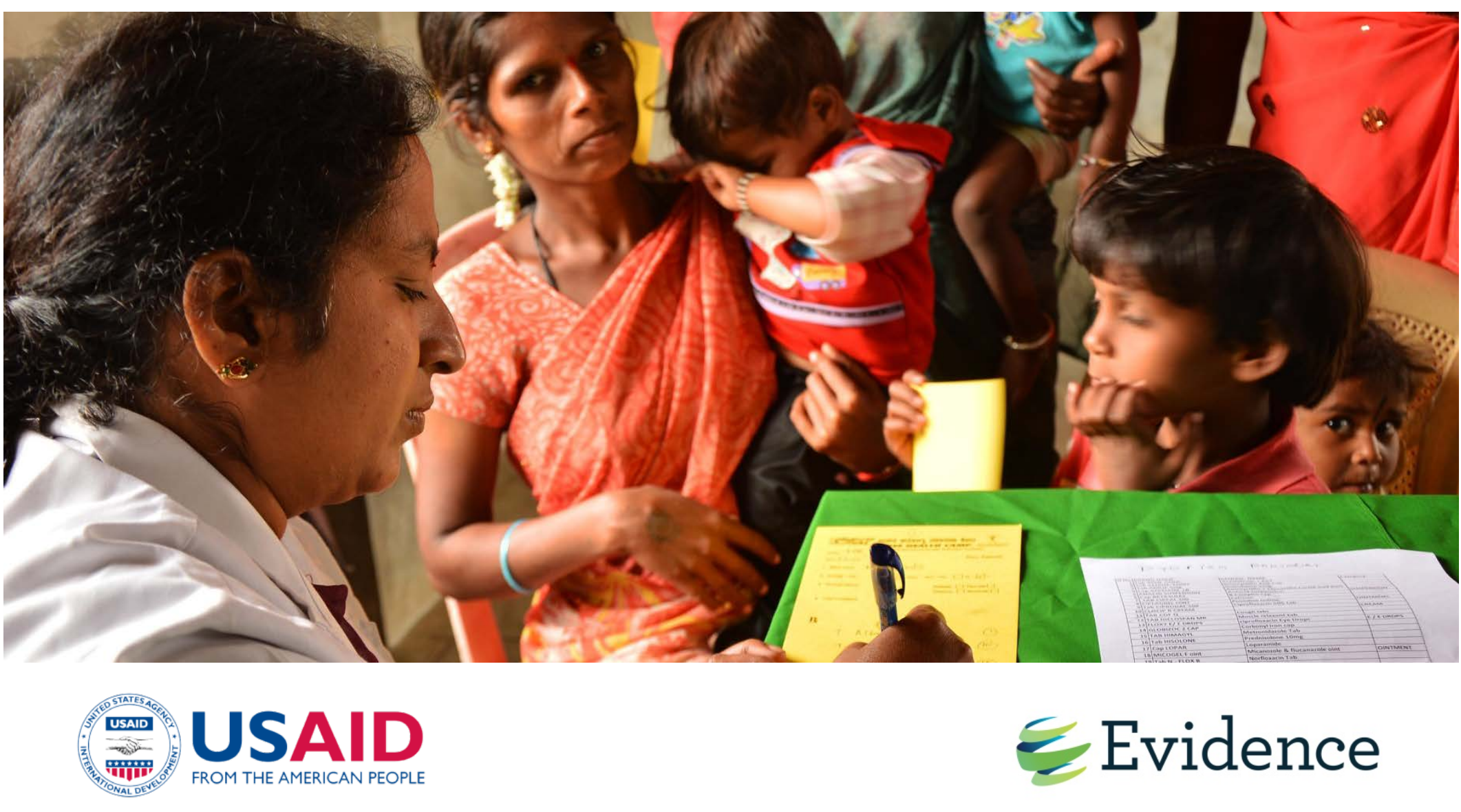





\section{Expanding the Role of Research Evidence in Family Planning Policy, Program, and Practice Decision-making}

Karen Hardee, Senior Associate \& Project Director, Evidence Project, Population Council Kelsey Wright, Staff Associate, Evidence Project, Population Council 


\section{The Evidence Project}

Population Council

4301 Connecticut Avenue, NW, Suite 280

Washington, DC 20008 USA

tel +12022379400

evidenceproject.popcouncil.org

F 1 The Evidence Project is made possible by the generous support of the (1) American people through the United States Agency for International Development (USAID) under the terms of cooperative agreement no. AID-

OAA-A-13-00087. The contents of this document are the sole responsibility of the Evidence Project and Population Council and do not necessarily reflect the views of USAID or the United States Government.

\section{Evidence}

The Evidence Project uses implementation science-the strategic generation, translation, and use of evidence - to strengthen and scale up family planning and reproductive health programs to reduce unintended pregnancies worldwide. The Evidence Project is led by the Population Council in partnership with INDEPTH Network, International Planned Parenthood Federation, Management Sciences for Health, PATH, Population Reference Bureau, and a University Research Network.

Published in September 2015

Suggested citation: Hardee, Karen and Kelsey Wright. 2015. "Expanding the Role of Evidence in Family Planning, Program, and Practice Decision-making," Working Paper. Washington, DC: Population Council, The Evidence Project.

Photo credit on cover page Dr. Tony Thomas, Trinity Care Foundation; Courtesy of Photoshare.

(C) 2015 The Population Council, Inc. 


\section{Table of Contents}

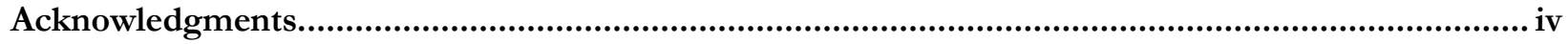

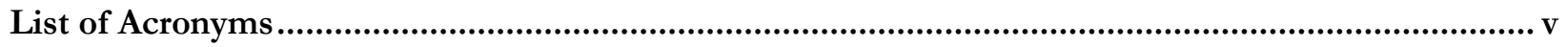

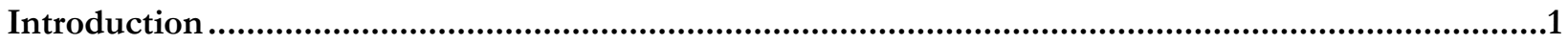

What is Evidence and How is it Used in Decision-making? ............................................................... 2

Promising Interventions to Expand the Role of Evidence in Decision-making................................. 3

1. Build Cultures of Evidence Use................................................................................................................. 3

2. Ground Research in an Understanding of Health Systems...........................................................................11

3. Build Research Utilization into Study Protocols...................................................................................... 12

4. Expand the Range of Research Methodologies for Studying Complex Health Systems ............................13

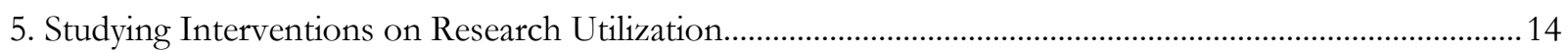

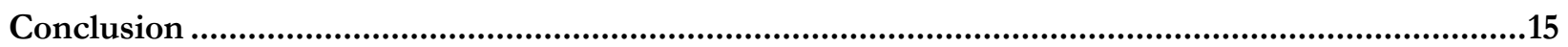

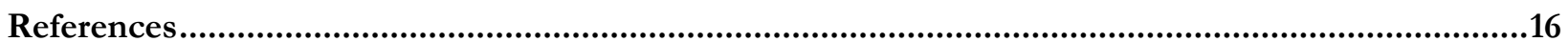




\section{Acknowledgments}

This publication was authored by Karen Hardee and Kelsey Wright of the Evidence Project. We are grateful to Rachel Sullivan Robinson, Associate Professor, School of International Service, American University, for helpful comments on an earlier draft of the paper. We also appreciate comments from a seminar at the Bill and Melinda Gates Institute for Population and Reproductive Health of the Johns Hopkins Bloomberg School of Public Health, including from Duff Gillespie, Professor, and Beth Frederick, Director of Advance Family Planning, among others. Comments from colleagues at USAID, including Mihira Karra, Erika Martin, Shawn Malarcher and others, also strengthened the paper. Many thanks as well go to Ellen Weiss of the Evidence Project for her edits, ideas, and family planning communications expertise and to Anneka Van Scoyoc of the Evidence Project for making the report, images, and appendices so visually effective. 


\section{List of Acronyms}

$\begin{array}{ll}\text { AFP } & \text { Advance Family Planning } \\ \text { CIP } & \text { Costed Implementation Plan } \\ \text { CSO } & \text { Community service organization } \\ \text { DFID } & \text { Department for International Development (UK) } \\ \text { GRIPP } & \text { Getting Research into Policy and Practice } \\ \text { HIP } & \text { High Impact Practices } \\ \text { IKT } & \text { Integrated Knowledge Translation } \\ \text { IBP } & \text { Implementing Best Practices } \\ \text { K4H } & \text { K4Health } \\ \text { MIS } & \text { Management information system } \\ \text { MOH } & \text { Ministry of Health } \\ \text { OR } & \text { Operations research } \\ \text { WHO } & \text { World Health Organization }\end{array}$




\section{Introduction}

Family planning's global resurgence over the past decade has increased attention on implementing policies and programs that are based on scientific evidence. The FP2020 initiative, which challenges countries and donors to expand access to voluntary family planning to an additional 120 million contraceptive users by 2020, has been particularly instrumental in promoting evidence-based family planning programming and policymaking. A focus on evidence-based decision-making in family planning policies, programs and practices is increasingly important as global development resources shrink. Ensuring that family planning policies, programs and practices are evidence-based requires the generation of evidence about what interventions work and how they can work better, and getting the evidence to be used by decision-makers.

The international family planning field was founded on research, including through demonstration projects, national surveys, and decades of operations research (OR) and now implementation science, in addition to special studies. Furthermore, attention to getting evidence into action, or research utilization, also spans decades in family planning (Freedman and Berelson 1976; Foreit and Frejka 1998; Seidman and Horn 1991; Simmons et al. 2002; WHO 2006). Current initiatives such as Family Planning High Impact Practices (FP HIP), the Implementing Best Practices (IBP) Initiative, K4Health and FP2020 all focus on promoting evidence-based family planning programming. With this rich history, there is surprisingly scant research on whether and how evidence is used in decision-making for family planning programming, policies, and practices, despite a growing literature on research utilization, also known as knowledge translation among other names, in global health (McKibbon et al. 2010; Moat and David 2012; Damschroder et al. 2009).

This paper focuses on the emerging knowledge translation literature to provide lessons learned on ways to increase the role that evidence plays in decision-making for family planning and reproductive health policies, programs, and practices. It is part of a larger synthesis of the literature that explores what "evidence" means, what types of evidence policymakers, program managers, and implementers seek when facing implementation decisions and how evidence relates to other factors that influence decision-making (Hardee et al. forthcoming).

This paper and the larger synthesis are based on a literature search whose objective was to locate references on evidence-based policy in health or social science, knowledge transfer, translation or utilization for health or social science programs, evidence collected from "intervention studies" in family planning programs, and "implementation science" for family planning and reproductive health. The literature search included databases, individual websites, and reviews of bibliographies. The search was conducted in PubMed/MEDLINE, Web of Science and POPLINE, in addition to snowballing references from bibliographies. References were limited to 2000-2014 in the database searches; snowballing of materials goes back to the $1990 \mathrm{~s}$, and in some cases earlier, in order to capture earlier literature on research utilization in family planning and seminal writing on research utilization. 


\section{What is Evidence and How is it Used in Decision- making?}

Researchers and decision-makers have different views of what constitute evidence for decision-making and the value of the various forms of evidence (Sumner et al. 2011; Brownson et al. 2009; Lomas et al. 2005; Lewis 2007; Bowen and Zwi 2005). Researchers consider evidence to be findings from research studies (with differences of opinion among researchers about legitimate methodologies) (STEP UP Research Programme Consortium 2013), whereas decision-makers consider a range of research studies, along with monitoring and evaluation data, program reports, policy documents, community input, and professional experience as evidence. Availability of local evidence is important to national decision-makers.

Decision-makers tend to filter scientific evidence through other factors, including their beliefs about the issue and whether the evidence fits those beliefs and is useful (e.g. feasible, cost-effective, implementable, etc.);

"Researchers and decisionmakers have different views of what constitutes evidence for decision-making" their values surrounding the issue; and on political, economic, and social constraints (Fisher et al. 1991; Cookson 2005; Kim 2006; Eyben 2013; DFID 2014).

The facilitating factors and barriers to evidence use have been consistent over time and are similar for family planning as for other health areas (Oliver et al. 2014; Hyder et al. 2010; Brambila et al. 2007; Simmons et al. 2007; WHO 2006; Almeida and Basolo 2006; Innvaer et al. 2002; Askew et al. 2002; Simmons et al. 2002; Trostle et al. 1999; Koenig and Whittaker 1991). Evidence use in decision-making can be influenced by the availability of relevant evidence; the extent of collaboration between researchers and decision-makers; the clarity of the presented evidence; the timeliness of the evidence; decision-maker and researcher skills; the strength or weakness of relationships between decision-makers and researchers; and the costs of implementing research findings. Continued challenges to evidence use in decision-making stem from differences in the contexts and reward structures under which researchers and policymakers work, particularly the different timescales of research and decision-making. 


\section{Promising Interventions to Expand the Role of Evidence in Decision-making}

BOX 1

While it is unrealistic to assume that research evidence will or should be the only factor which influences decision-making (Kay 2010), it is important to ask the question of whether there are potential interventions that could increase the "space" that research evidence holds in the decision-making process. Based on the literature, we identify five categories of interventions that should be considered to enhance the contribution of research to decisions on family planning policies, programs, and practices (Box 1).
Interventions to Enhance the Contribution of Research to Family Planning and Reproductive Health Policy, Program, and Practice Decision-making

1. Build Cultures of Evidence Use

- Building Relationships: Engaging Decision-makers and Researchers in Evidence Generation and Use

- Building Capacity: Strengthening Capacity for Evidence Use

- Using Intermediaries Between Researchers and Decision-makers

- Building Knowledge Translation Platforms

- Supporting Rapid Response Mechanisms to Provide Evidence

- Making Research Directly Available

- Better Packaging and Communication of Findings

2. Ground Research in an Understanding of the Health System

3. Build Research Utilization into Study Protocols

4. Enhance Research Methodologies for Studying Complex Health Systems Issues

5. Study Interventions on Research Utilization

\section{BUILD CULTURES OF EVIDENCE USE}

Many researchers and international agencies have made calls for fostering cultures of using evidence in decision-making (Askew et al. 2002; WHO 2004; Lewis 2007; Dopson 2010; Hyder et al. 2010; Koon et al. 2013; Nutley and Reynolds 2013; LaPelle et al. 2014; Armstrong et al. 2014). This entails creating a common understanding among both decision-makers and researchers of concepts like "evidence use," "research utilization," and "decision-making." It additionally includes engaging decision-makers in research development from the outset-this allows for engagement and investment in the research and its results. Additionally, supporting the training and capacity building of researchers to understand the processes and contexts in which decision-making, policies, and programs occur is essential to enable researchers to be effective brokers of their research results and to ensure that researchers are designing research that has value to decision-makers. Additional research is needed to identify the best timing, mechanisms, and forums for doing capacity building with both researchers and decision-makers. Finally, it is essential that decision-makers have access to existing and forthcoming evidence, particularly evidence that is related to key decisions that are within their purview. Recent initiatives by global donors to ensure that funded research results (both publications and datasets) are open access are examples of encouraging progress in this area. Strategies to enhance cultures of evidence are discussed in more detail below: 


\section{Building Relationships: Engaging Decision-makers and Researchers in Evidence Generation and Use}

One way to build a culture of evidence use is to foster ties and strengthen understanding between researchers and decision-makers. Rather than viewing 'decision-making' and 'research' as product-centered, moving toward a mutual understanding that both decision-making and research are processes-each has different time frames, requirements, and incentives-would be beneficial in bridging the gap between the two groups (Lomas 1997).

Family planning OR programs have consistently highlighted the need to engage decision-makers early in the research process. These decision-makers should come from all levels of the health system where decisions are being made about policies, programs, or practices. Active engagement of users from the beginning of the research process increases the chances that study results will be used (Seidman and Horn 1991; Koenig and Whittaker 1991; Fisher et al. 1991; Solo et al. 1998; Ross 1998; Askew et al. 2002; Nath 2007; FRONTIERS NDa; Sumner et al. 2011). This engagement can help ensure that the recommendations are feasible and implementable within the health system (FRONTIERS NDa). In addition to including decision-makers in defining the research and shaping key interventions, Koenig and Whittaker (1991) and Kim (2006) note the utility of taking decision-makers to OR sites to demonstrate the value and feasibility of interventions.

One of the current models for decision-maker involvement in research promotes a more active role for decision-makers in the research process, including as partners in implementing research. Sometimes called integrated knowledge translation (IKT), this model is increasingly promoted by funders and organizations, including the Alliance for Health Policy and Systems Research, which has issued recent calls for decisionmaker-led implementation research (www.who.int/alliance-hpsr/). The assumption behind IKT is that research will be more useful for policies and programs if implementers are more actively involved in the design of studies. Kothari and Wathen (2013) note the need to examine the assumptions behind IKT, namely that the onus of responsibility for reaching out to decision-makers to develop partnerships falls on researchers; that resources exist to support these partnerships; that the effort to develop and sustain these partnerships is worthwhile despite the lack of institutional incentives for both researchers and decisionmakers; and that the evidence that results from this 'jointly implemented' research is more actionable than research undertaken by researchers alone. The need for professional incentives for both decision-makers and researchers to sustain such partnerships has also been highlighted by others (Kerner 2008; DFID 2014; Kothari and Wathen 2013). Furthermore, Freudenberg and Tsui (2014) contend that partnerships forged by researchers with policymakers can be dominated by judgments that value evidence over the messy world of politics and power in which policy and program decisions are made. They note the need to include in these partnerships stakeholders with the ability to work across the research and policy/program decision-making worlds (see the section on using intermediaries below).

\section{Building Capacity: Strengthening Capacity for Evidence Use}

The lack of capacity of policymakers, program managers and implementers to use evidence in their decisionmaking has long been cited as a constraint in many studies and reviews (Koenig and Whittker 1991; DFID 2014; Nutley and Reynolds 2013; Hyder et al. 2010; Ongolo-Zogo et al. 2015). In 2014, a review by DFID examined a number of studies that addressed the capacity to use research in low- and middle-income countries and concluded that "unless there is sufficient capacity to absorb research results, no amount of 
research supply will have positive impacts" (DFID, 2014: 43). This constraint holds true in high-income contexts as well: Law (2010: 107) notes that "on the health system side, we have only begun to scratch the surface in Canada with respect to equipping individual decision-makers with the tools, skills, and experience necessary to engage the 'pull' side of research use effectively." Part of the issue in low- and middle-income countries is lack of human capacity in health systems that are stretched thin (Alvaro et al. 2010; DFID 2014). This does not imply that decision-makers themselves necessarily need to be trained, but that those decisionmakers should be surrounded by and supported by advisors who are trained and competent in understanding evidence based decision-making. To this end, DFID supports the BCure initiative that links projects across Africa and South Asia to

“...decision-makers should be surrounded by and supported by advisors who are trained and competent in understanding evidence based
decision-making." build policymakers' and practitioners' capacity to assess and use rigorous research evidence in their decision-making (https://bcureglobal.wordpress.com/). Ashford et al. (2006) describe a successful initiative that enhanced the use of evidence in decentralized health plans in Kenya, noting that "it was important for district staff to see the links between the priority needs identified in the surveys and the activities they prepared plans for, because it gave credibility to the ministry's reform planning effort" (Ashford et al. 2006: 670). There is need for additional research and focus on improving the capacity of decision-makers to understand and use research, including identifying strong and weak research. Topics for this research could include the timing of trainings or orientations for decision-makers, and mechanisms of training (e.g. what formats are most effective at promoting evidence use). Ashford et al. (2006) also recommend enhancing the use of evidence by integrating its use into the policy and program decision-making process linked with routine planning and management exercises.

\section{Using Intermediaries between Researchers and Decision-makers}

Given the complexity of the policy and program decision-making environment, including for implementers deciding to implement evidence-based practices, the lack of understanding between researchers and decisionmakers, and the lack of incentive structures, evidence suggests that many researchers may not be the best messengers of their research (Askew et al. 2002). This is in part because they are not trained to engage in the decision-making process or in program implementation in ways that could support use of their research (Trostle 2006).

The importance of having intermediaries_also referred to as knowledge brokers, mediators, policy champions or policy entrepreneurs-between researchers and decision-makers is not new in family planning. In 1998, Ross wrote about the importance of intermediaries in family planning OR, saying that "Managers...may listen to...key persons located in the interface between the researcher and the manager" (Ross 1998: xii). Askew et al. (2002: 6) wrote that, "A range of other actors, some of which are advocates for a particular moral or policy position, often mediate [the] relationship." Brambila et al. (2007) highlighted the need for champions to promote the expansion of evidence-based interventions. FHI successfully supported 
the use of champions "to help bridge the gap between research and programs" related to family planning and HIV integration in Nigeria, Tanzania, Uganda, and Zambia (FHI 2008a). As intermediaries, Advance Family Planning (AFP) has worked with other partners to undertake successful evidence-based advocacy to promote family planning in the Democratic Republic of Congo, Kenya, Sengal, Tanzania, Uganda, India, and Indonesia, and with the United Nations Commission on Population and Development. From efforts, AFP has documented increased political and resource commitments for family planning at global, national and decentralized levels and revisions to policies (APF 2015a,b,c,d,e,f,g,h). Although it does not specify what evidence, among other factors, was persuasive, AFP stresses the importance of evidence-based messages and packaging data for decision-makers in simple language with specific "asks" in the local langauges of the decision-makers.

Knowledge brokers can come from a range of organizations. These individuals or groups "will make the most of networks but will also use connections or negotiating skills, be persistent, develop ideas, proposals and expertise well in advance of policy 'windows' - whether brought about because of a change in government, citizen action, or a swing in the national mood" (Neilson 2001, cited in Crewe and Young 2002: 29). While researchers may be one group that decision-makers consult, credible advocates for policy and program decision-making may also consult with ministries of health and other sectors, donors, research institutions, non-governmental organizations, women's groups and political, religious, and community leaders, which can increase their credibility and visibility around specific issues (Yamey and Feachem 2011; Smith et al. 2015; Haynes et al. 2012; Brambila et al. 2007). From decision-makers' perspective, credibility of evidence is often determined based on whom the decision-makers are deemed "trustworthy" (Haynes et al. 2012), rather than who may or not be the most knowledgeable on the research. Writing about use of evidence for post-abortion care in two countries in West Africa, Askew (2006: 90) noted that policymakers in ministries said they were most likely to consider evidence if it was presented to them by some they knew personally and considered to be credible. Knowledge brokers need to be supported by staff who can review data and evidence for relevant themes and messages that can be tailored to the stakeholders who will be making decisions (Lavis 2006).

Some authors question the role of intermediaries, saying that knowledge brokering may be more successful to inform clinical practice than complex policymaking. As noted by Fox, "Policymakers do not look around for a broker when they are under pressure to make a decision, which they always are. They look to their staff who either know or do not know how to find and use the best available evidence" (Fox, 2004, cited in WHO, 2004: 103). Engaging the staff of decision-makers as intermediaries may be an effective strategy to ensure evidence-informed decision-making.

\section{Building Knowledge Translation Platforms}

There is currently a strong push in global health to promote knowledge translation platforms that link producers of research to users of research by making evidence syntheses available, and by developing demand for research and facilitating deliberative dialogues that bring stakeholders together to discuss bodies of research (Moat et al. 2014). Knowledge translation platforms are operational in 12 countries in sub-Saharan Africa "as partnerships among health stakeholders (policymakers, researchers, civil society, and media) to promote the systematic use of research evidence in policymaking about health systems through the production and dissemination of targeted evidence syntheses, the organization of evidence informed deliberations on health priorities, and capacity building of stakeholders" (Ongolo-Zogo et al. 2015: 3). This combination of evidence briefs (based on systematic reviews) and deliberative dialogues seeks to address the 
complaint that research is not relevant or easy to use in decision-making. Neither the production of evidence briefs nor the use of platforms (e.g. family planning technical working groups or fora to develop national plans or operational strategies) is new to family planning. The current strategy of getting evidence producers and users together to determine the recommendations from research has been recommended since the early days of OR (Fisher et al. 1991).

A review of use of evidence briefs and deliberative dialogues in Burkina Faso, Cameroon, Ethiopia, and Nigeria found that use of both of these methods increased policymakers' intention to use evidence (Moat et al. 2014). Additional research is needed to understand policymakers' actual use of evidence following interventions such as deliberative dialogues and policy briefs, and whether knowledge translation platforms will be successful in promoting use of research evidence in decision-making (DFID, 2014). One systematic review did not find that interventions to increase policymakers' access to systematic reviews led to measurable increases in use of the evidence (Murthy et al. 2012).

The USAID-funded K4Health $(\mathrm{K} 4 \mathrm{H})$ website provides a platform for evidence-informed family planning and reproductive health programming (www.K4H.org). The IBP initiative "is dedicated to strengthening the capacity of the family planning community to identify, implement, and scale-up effective practices through sharing knowledge and resources" (http://www.ibpinitiative.org/). The Family Planning High Impact Practices (HIP) initiative provides evidence from HIP briefs on "effective service delivery... or systems interventions that when scaled up and institutionalized, will maximize investments in a comprehensive family planning strategy" (https://www.fphighimpactpractices.org/). Assessments of the use of the family planning service delivery HIPs are being undertaken by the IBP HIPs Task Team in Mozambique, Tanzania, and Guatemala. FP2020's website includes a knowledge platform designed for “connecting people, ideas, and evidence to inspire, inform, and accelerate progress for family planning" to achieve the goal set out at the 2012 London Family Planning Summit of reaching an additional 120 new family planning users by 2020 (www.familyplanning2020.org).

Linking family planning-specific initiatives, such as FP2020, IBP, and the Family Planning HIP Initiative, with the more general evidence use community, could potentially result in extending the scope and understanding of existing evidence use initiatives while allowing the family planning field to capitalize on the extensive experience and knowledge that currently exists. Regional and local organizations to promote evidenceinformed policy and program decision-making are emerging. For example, the Regional Center for Quality of Health Care at Makarere University in Uganda is a clearinghouse for evidence-based information concerning quality improvements in reproductive health (www.rcqhc.org) and the African Institute for Development Policy links demographic and other research with policy and practice (http://www.afidep.org/).

Sustainability is a huge issue with these initiatives, given that they are often funded by donors and that this funding may not continue to be available once the project funding has ended. One such initiative related to family planning was the Getting Research into Policy and Practice (GRIPP) initiative funded by DFID—to build an online evidence base of case studies documenting the activities undertaken to promote utilization of research findings. The website was closed down when donor funding ended and these materials are no longer available (Nath 2007), although the papers presented at one conference are still online (http://www.socstats.soton.ac.uk/choices/workshop/). Having a repository for all such "orphaned," but still useful, evidence related to family planning is needed, perhaps through the $\mathrm{K} 4 \mathrm{H}$ platform (https://www.k4health.org/). 


\section{Supporting Rapid Response Mechanisms for Providing Evidence}

Efforts to establish regional and national rapid response mechanisms to meet the evidence needs of decisionmakers in a timely way have yielded some successes. For example, a rapid response mechanism based at Makerere University in Uganda and supported by a national, regional, and global network of researchers was coordinated by hired staff that kept in regular contact with policymakers and health systems' stakeholders $(\mathrm{MOH}$, districts, CSOs, health-related multi- and bi-lateral agencies and the private sector) (Mijumbi et al. 2014). These staff interacted with the policymakers and decision-makers to ensure that their questions could be answered within one month. The users of this rapid response mechanism tended to be mid-level policymakers at the $\mathrm{MOH}$, with no one from the districts using the service. Interestingly, the study found that the service did help the recipients. In 30/65 cases (46 percent), the decision-maker changed their course of action after receiving assistance from the rapid response mechanism. The service gave them more options to consider and there was high satisfaction with the service. "Policymakers echoed what several researchers have found: that policymakers are indeed interested in using research evidence and do value what it contributes to the policymaking process" (Mijumbi et al. 2014: 13).

Rapid response mechanisms have challenges. An assessment of the feasibility of establishing a regional mechanism in South East Asia, which would have included on-call mechanisms, information centers, knowledge networks, and centers for systematic reviews, generated interest among stakeholders. The assessment noted four key challenges: research capacity, resources (sustainability), diversity of countries/languages, and responsiveness (Healy et al. 2007). The assessment concluded that "moving from the broad idea of an Asian regional mechanism to action will require ongoing consultation, detailed planning, and phased implementation" (Healy et al., 2007: 1-5). The rapid response mechanism in Uganda faced both demand and supply challenges. Decision-makers were skeptical that the researchers would understand their needs, but ended up referring the service to colleagues. Makarere University had trouble finding staff with the right skills to find and synthesize evidence for the decision-makers in a short timeframe. They also noted the need for fast and reliable internet access to be able to access materials, in addition to the need to be able to access full text research papers. Finally, they noted the need to focus on sustainability once donor funding ended (Mijumbi et al. 2014).

\section{Making Research Directly Available}

Access to peer-reviewed published evidence has historically been limited to subscription-based or academicbased access; however, there are a number of initiatives underway to enhance global access to evidence, including increased donor focus on making their funded results publicly available. Researchers or program implementers who receive foundation or government-based funding are increasingly being required to make not only their publications, but also

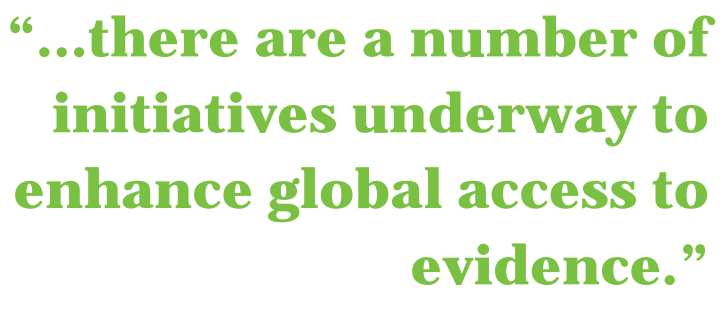
their research data sets, available via open access journals and databases. This requirement allows for a democratization of evidence as access to this information and knowledge is now globally possible with only an internet access point. Despite this increasing requirement, not all research is available via open access journals. Researchers who are publishing evidence 
that has the potential to be useful to decision-making for policies, programs or practices should consider submitting their materials to open access journals.

Making evidence available that may not traditionally be perceived as evidence, such as documentation of implementation, and translating a policy into practice, is something that should be encouraged. These types of evidence are essential for ensuring that the existing literature is not utilized in isolation but continues to be built upon as the evidence expands and grows. The Development Experience Clearinghouse that provides information from USAID projects spaning half a century is an example of making programmatic evidence available (https://dec.usaid.gov/dec/about.aspx). Additionally, continued focus on publishing findings and making them widely available allows for the dissemination of evidence between countries and globally to share and learn from similar issues and to create context-informed and evidence-based solutions.

An initiative in the US to make evidence more directly available to state-level public health department practitioners through electronic access to journals found that the practitioners appreciated the access but that they were short staffed and most "advocated restoring a culture of evidence-based practice by identifying appropriate committed [public health department] staff with the authority to champion evidence-based practice and usage of digital library resources" (LaPelle et al. 2014: 78). Creating supportive environments where increased accessibility to evidence is coupled with the institutionalization of evidence champions is also important for improved evidence uptake-without purveyors and translators of research findings, the increased accessibility of evidence may well fall flat in its efforts to influence evidence-informed decisionmaking.

\section{Better Packaging and Communication of Findings}

The critical importance of communicating research findings to a range of audiences is clearly noted in the literature (MEASURE Evaluation 2009). Long research reports, while suitable for reaching other researchers, are not appropriate for communicating with decision-makers. There is a strong negative association between the complexity of research findings (or of their presentation) and utilization of these findings, and between the use of academic jargon and the likelihood of evidence being used by policymakers (Dobbins 2002; Oxman et al. 2009b). Providing different stakeholder groups with the evidence they need, in technical and national languages they can understand and in ways that are respectful to them is part of the communication process (Ulin et al. 2005). The way in which findings are communicated to decision-makers can make the difference between evidence being ignored or being used for decision-making. For example, the way in which research on sensitive issues is handled, particularly research on a social, religious, or cultural topic, could determine the extent to which the research results are accepted and used on a wider scale (Nath 2007). Communicating results with stories can help "frame scientific facts in meaningful ways" (Hillsman 2015).

Promoting decision-maker literacy in research findings through ongoing collaborations and trainings with researchers has the potential to go a long way in terms of increasing evidence use and in terms of helping researchers to identify priority evidence and syntheses needed by decision-makers. An additional issue that is repeated throughout the literature is the need to assist decision-makers with the distinction between "no effects" and "negative effects" within research—often findings where there are "no effects" are interpreted as being negative effects, which can influence decision-makers to have negative perceptions of these findings rather than supporting additional investigations or evidence (Oxman et al. 2009e). 
Communicating findings clearly while taking into consideration the timing, policy, and budget context is important for encouraging increased and improved use of evidence in decision-making (Spicer et al. 2014). Analyzing the values, beliefs, and socio-political contexts in which key stakeholders operate can help researchers and knowledge brokers to identify strategies for presenting data to the decision-makers who have the most invested or the most influence on the issue at hand (Nath 2007). Researchers might consider partnering with advocacy organizations to communicate research findings to decision-makers to ensure that messages are tailored to various audiences (Brownson et al. 2009). For example, Advance Family Planning, an advocacy initiative that builds on the momentum of the 2012 London Summit on Family Planning, focuses on providing decision-makers with evidence that family planning is a sound investment with dividends in health and women's empowerment, socioeconomic development, the environment, and other areas (www.advancefamilyplanning.org $/$ ). Researchers can also benefit from training on communicating their findings to various audiences. The Population Reference Bureau has long supported policy communication workshops and other training for researchers and journalists to improve the way evidence is shared with policymakers. The title of one such presentation was "Communicating Research to Policymakers: The Road to Inaction is Paved with Research Reports" (Ashford 2001).

Enhancing the availability of evidence will be necessary but not sufficient to improve evidence-based programming and policies unless mechanisms are put in place to create "evidence literacy" for nonresearchers or to create mechanisms that rapidly synthesize evidence for use by decision-makers. For

"...policymakers preferred to receive both abridged and fulllength materials, usually in the form of a one to three-page brief and a longer report." example, Rosenbaum et al. (2011) carried out 21 user tests in six low- and middleincome countries to test users' experiences with evidence brief formats and to generate evidence on using evidence briefs. They found that policymakers preferred to receive both abridged and full-length materials, usually in the form of a one to three-page brief and a longer report on the research

(Rosenbaum et al. 2011). Additionally, they particularly valued sections of the briefs that explained the relevance for low- and middle-income countries and perceived these sections to compensate for the lack of locally relevant detail in the original evidence (Rosenbaum et al. 2011). Some decision-makers struggled with understanding the numbers and text and had a poor understanding of what a systematic review was. Additionally, participants often expected information to be included that wasn't, such as recommendations, outcome measures, or costs, and many stated that they wanted shorter, clearer summaries (Rosenbaum et al. 2011). This evidence demonstrates the need to make additional kinds of evidence available, while also focusing on increasing the availability of easy-to-understand formats for evidence dissemination and improving the skills of end-users of evidence. 


\section{GROUND RESEARCH IN AN UNDERSTANDING OF HEALTH SYSTEMS}

Health systems are complex systems; research on family planning where the findings are intended to be used in programming needs to reflect that complexity. Countries generally have multiple ministries, departments, and organizations involved in implementing policies and programs. Sometimes lines of authority for making changes and for allocating resources are not clear. Using the United States as an example, Kerner (2008) illustrates within the public sector, at the federal and state level, why it is difficult to get research into practice (Figure 1). "How best to integrate science into the program and policy decision-making that takes place in this complex system is not obvious, nor easy to evaluate" (Kerner 2008: 196). Research that bypasses the operation of health systems by setting up special circumstances such as adding additional staff or instituting new management information system (MIS) forms, for example, may or may not be feasible to implement in the program after the study is completed (Cross et al. 2001). A feasibility study of the integration of family planning and STI services in Jamaica found confusion around whether authorities at the local, regional, or national level had the jurisdiction to make policy, program, and resource allocation decisions about the integration initiative (Policy Project 2005). Amin et al (2007) demonstrated in a review of changing the national malaria drug policy in Kenya to provide artemisinin-based combinations that the process of changing practices to integrate new therapies is incredibly complex, from a financial, political, legislative, and organizational perspective. The theory of path dependence suggests that incremental changes will be easier to implement than large changes and that those responsible for implementing the changes will interpret the changes to make them fit existing systems and processes (Torfing et al. 2009).

A number of studies have noted the need to take advantage of fortuitous timing when promoting the use of evidence in decision-making (Brambila et al. 2007; Freudenberg and Tsui 2014). Making sure that research is timed to be useful for program planning and budgeting cycles is important (Seidman and Horn 1991; Haaga and Maru 1996; Spicer et al. 2014). Writing about use of evidence for post-abortion care in two countries in West Africa, Askew (2006: 90) noted that policymakers in the ministries "emphasized that recommendations from research should: i) address existing $\mathrm{MOH}$ priorities; (ii) be presented clearly so that they could be understood by non-researchers; and (iii) be timed to coincide with planning and budgeting cycles." The advent of widespread family planning Costed Implementation Plans (CIPs) following the 2012 London Summit on Family Planning provides a timely opportunity for researchers and decision-makers to collaborate to review national family planning policies and strategies and to conduct research on the extent and types of programming that have been conducted under these CIPs. This type of evidence production aligns with

country-determined plans while providing documentation of processes that have regional and global relevance. 
Researchers who implement research intended to affect policies, programs, and practices and be implemented within health systems, would benefit from training in navigating the tensions between politics and science and in the tools of advocacy and participation (Freudenberg and Tsui 2014: 13). Understanding the range of

\section{Executive Branch Departments and CONGREssional Committees That Control Programs for Children and Families AND HOW THEY RELATE TO EACH OTHER}

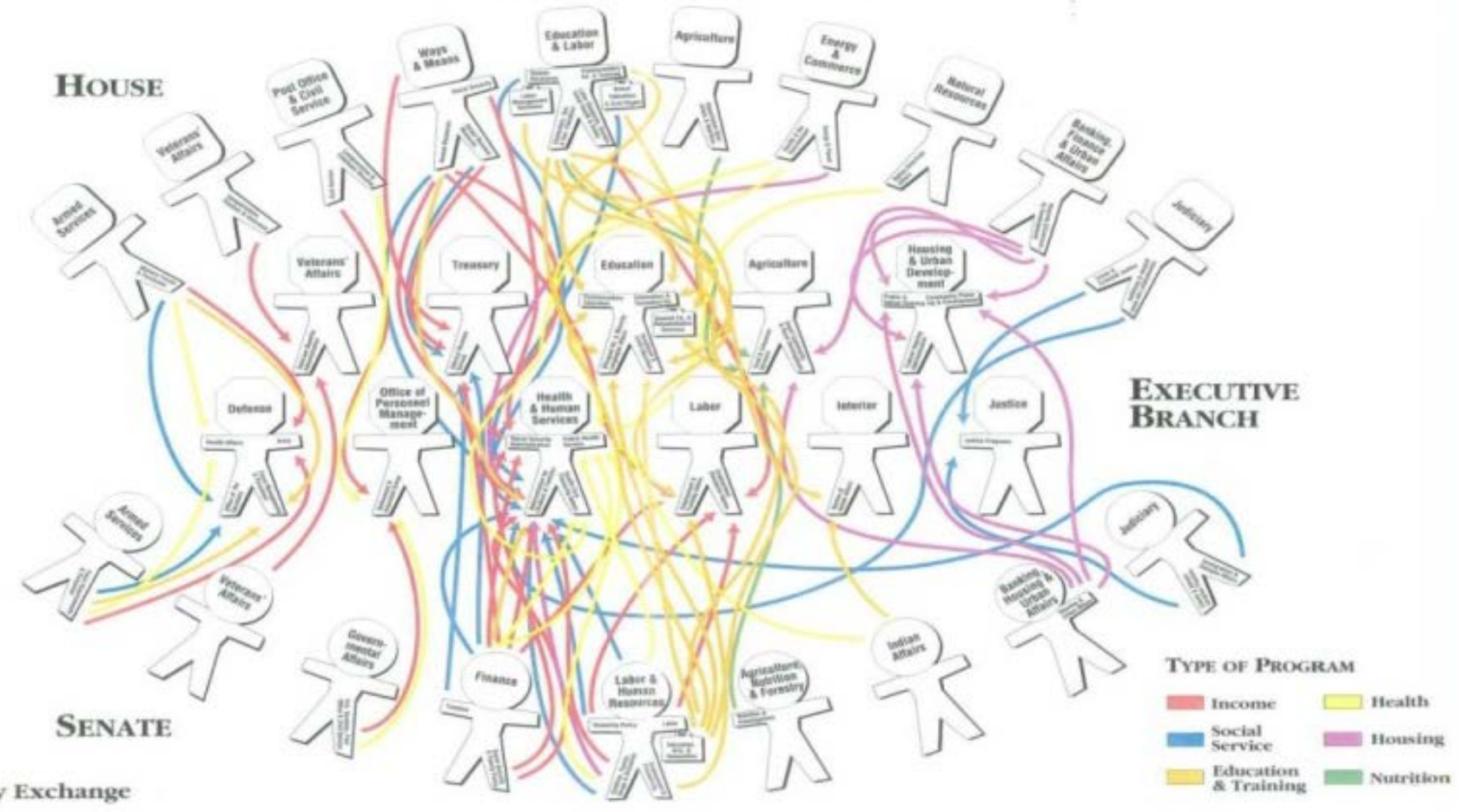

The IEL. Policy Exchange

factors that mediate the use of evidence in decision-making, and understanding the positions and views of decision-makers is important. Furthermore, how complex and continuous an issue is and the availability and certainty of the evidence about the issue and its feasibility of implementation in the health system, are all important for researchers to determine as they consider their research and increasing its utility for decisionmaking.

\section{FIGURE 1}

\section{BUILD RESEARCH UTILIZATION INTO STUDY PROTOCOLS}

Research protocols generally have sections on dissemination that follow a push model of information sharing by publishing a report, holding a dissemination meeting, or developing a journal article. This model is geared more toward the needs of researchers for publication than for decision-makers' needs for decision-making. While these steps are important, enhanced research utilization sections of protocols can ensure that activities which identify the policy or program stakeholders who are important to the research will be conducted. 
Additionally, enhanced research utilization sections in protocols can help define how key stakeholders will be included during the course of the study, what policy or program decisions the study might be useful for, and anticipated actions of the study team to promote utilization of the findings. This section does not imply that the study team will be doing the research utilization themselves, but shows what they will do to link with others to enhance the potential for use of the study findings.

\section{EXPAND THE RANGE OF RESEARCH METHODOLOGIES FOR STUDYING COMPLEX HEALTH SYSTEMS}

The evidence-based medicine framework that favors the randomized control trial methodology to examine a fixed intervention is increasingly recognized as an inappropriate tool for measurement of complex health systems. Nevertheless, "there is a strong temptation to apply evidence-based medicine methods and standards reflexively to public health" (Shelton 2014: 253). Increasingly, this has resulted in a demand for multifaceted approaches and research designs that allow for the examination of interventions in complex, real-world health systems (Almeida and Bascolo 2006; Askew et al. 2002; Behague et al. 2009; McCoy et al. 2010; Braveman et al. 2011; Brownson et al. 2009; Lavis et al. 2012; Simmons et al. 2002; Simmons et al. 2007; Yamey and Feachem 2011; Eyben et al. 2013; and Clar et al, 2011). There are increasing calls for the use of a range of research methods to study reproductive health programming; these methods may fall outside historical definitions of rigorous evidence; however, they may have more to contribute to policy and decision-making processes (STEP UP 2013). Studies that include information on costs and other resource needs along with evidence for change compared to current programming can enhance their usefulness in decision-making (Oxman et al. 2009b). Providing comparative options for decision-makers based on clear distinctions between resource needs and outcomes has also been found to be useful to decision-makers (Oxman et al. 2009a). The rise of

"There are increasing calls for the use of a range of research methods to study reproductive health programming." health policy and systems research, implementation science, and attention to studying complex adaptive systems, along with the growth of journals that publish findings from these types of studies, are responses to this need to focus research on health systems and improving the development and implementation of policies and programs (Panisset et al. 2012; Sheikh et al. 2014; USAID ND; University of Cambridge 2014; Paina and Peters 2011).

Methodological criteria for syntheses of the literature should also include a wider range of evidence than randomized control trials as "strong" evidence. These criteria should include the gray literature on programs as critical pieces of evidence for triangulation with published outcomes on evidence. How an intervention is implemented, in addition to the outcomes of the intervention, are both important for decision-making. FHI 360 has developed a tool to assess the divergence between the intervention elements as intended and as implemented in studies, with an illustration of its use in a study of expanding contraceptive options for clients of prevention of mother-to-child HIV transmission services in South Africa (Hoke et al. 2014). 


\section{STUDYING INTERVENTIONS ON RESEARCH UTILIZATION}

Studies of the decision-making environment and the role of evidence in decision-making are emerging for a number of global health areas, most notably HIV, malaria, and TB (Burris et al. 2011; Hutchison et al. 2011; Hunsmann 2012). Wathen et al. (2013) followed a particular study on screening for intimate partner violence to see where and how the study was cited, including in policies and practice guidelines. They found that evidence from the same study was not used consistently across the sources in which it was cited (Wathen et al. 2013). Oxman et al. (2009b) note a lack of research on improving the use of evidence in decision-making with regard to the following dimensions: the degree of involvement of policymakers, the different types of forums for communication, methods for recruiting stakeholders, and the best ways of training and supporting stakeholders to ensure effective involvement throughout the evidence generation and use process (Oxman et al. 2009b). Studies of how evidence is used in decision-making on family planning polices, programs, and practices, and how it is cited in policies and guidelines would be beneficial to improve evidence to action interventions.

As more research examining implementation and scale-up of evidence-informed family planning practices is undertaken, a focus of researchers should be to evaluate and share these efforts, as documentation of the process of using evidence in decision-making is scarce (Nutley and Reynolds 2013). A number of frameworks to study evidence use or research utilization are available (Damschroder et al. 2009; Glasgow and Emmons 2007; Bowen and Zwi 2005; Crewe and Young 2002; Brownson et al. 2009; DFID 2014), including three that focus specifically on family planning and reproductive health (Nath 2007; Bertrand and Marin 2001; Sumner et al. 2011). These frameworks assess a number of dimensions, including the context in which evidence is considered, the process of considering the evidence and the relationships among researchers and decisionmakers. 


\section{Conclusion}

This paper identifies five promising interventions that can increase the likelihood that decision-makers will include evidence among the factors that guide and influence their decisions. The first intervention focuses on building cultures of evidence through enabling relationships between the producers and users of evidence, through strengthening capacity to use evidence, and through developing mechanisms for knowledge transfer and communication. When evidence use in decision-making becomes normative, getting research included in decisions about policies, programs, and practices should be easier. Secondly, grounding research in an understanding of complex health systems will help ensure that the studies undertaken provide evidence that is more likely to be useful for programs and feasible to implement within health systems. Thirdly, researchers can expand "dissemination" sections in their protocols to focus on research utilization by linking the study with the health system context and identifying stakeholders and processes (e.g. budgeting and planning) that are important for potential use of the study findings. This does not imply that principal investigators of studies need to be the only ones to engage in research utilization; these sections can also identify stakeholders to be involved in promoting use of the evidence. Fourthly, the current discourse on standards of evidence (STEP UP Consortium 2013) and the need to move beyond context-free randomized control trials to strengthen research methodologies for studying complex health systems is closely linked with discussions of promoting evidence use in decision-making. Finally, in family planning and reproductive health, insufficient attention has been paid to studying interventions to increase the use of evidence in decision-making. This line of inquiry will enhance our efforts to increase the "space" that research evidence holds, among other legitimate evidence and factors, in the policy, program and practice decision-making process. 


\section{References}

Advance Family Planning. 2015a. "Democratic Republic of the Congo Commits to Family Planning Through the Global FP2020 Partnership." Case Study. www.advancefamilyplanning.org.

Advance Family Planning. 2015b. "Four Kenyan Counties Develop Costed Family Planning Strategies." Case Study. www.advancefamilyplanning.org.

Advance Family Planning. 2015c. "Mobilizing Mayoral Support in Senegal.” Case Study. www.advancefamilyplanning.org.

Advance Family Planning. 2015d. "Government of Tanzania Allocates 2 Billion Shillings to Family Planning for 2014-2015.” Case Study. www.advancefamilyplanning.org.

Advance Family Planning. 2015e. "Uganda National Conference on Family Planning Leads Districts to Renew Commitments." Case Study. www.advancefamilyplanning.org.

Advance Family Planning. 2015f. "Uttar Pradesh Initiates Review and Revision of Population Policy.” Case Study. www.advancefamilyplanning.org.

Advance Family Planning. 2015g. "Indonesia Advocacy Working Groups Revitalize Family Planning Efforts at the District Level." Case Study. www.advancefamilyplanning.org.

Advance Family Planning. 2015h. "Influencing the United Nations Commission on Population and Development Outcome." Case Study. www.advancefamilyplanning.org.

Almeida, C., and Bascolo, E. 2006. Use of research results in policy decision-making, formulation, and implementation: a review of the literature. Cad Saude Publica, 22 Suppl, S7-19; discussion S20-33.

Alvaro, C., R.F Lyons, G. Warner, S.E. Hobfoll, P.J. Martens, R. Labonté, and E.R. Brown. 2010. "Conservation of resources theory and research use in health systems." Implementation Science. 5:79.

Armstrong, R., E. Waters, L. Moore, M. Dobbins, T. Pettman, C. Burns, B. Swinburn, L. Anderson and M. Petticrew. 2014. "Understanding evidence: a statewide survey to explore evidence-informed public health decision-making in a local government setting." Implementation Science 2014, 9:188 doi:10.1186/s13012-014-0188-7.

Ashford, L. 2001. "Communicating Research to Policymakers: The road to inaction is paved with research reports." Presentation at the Moving Beyond Research to Influence Policy Workshop. University of Southampton, 23-24 January. Accessed on March 7, 2014 from http://www.socstats.soton.ac.uk/choices/workshop/ashford.html

Ashford, L., R.R. Smith, RM De Souza, F.F. Fikree, and N.V. Yinger. 2006. "Creating Windows of Opportunity for Policy Change: Incorporating Evidence into Decentralized Planning in Kenya." Bulletin of the World Health Organization. 84(8): 669-672.

Askew, I. 2006. "Using operations research to introduce postaboration care services in Burkina Faso and Senegal." In WHO. Department of Reproductive Health and Research. 2006. Turning Research into Practice: Suggested Actions from Case-studies of Sexual and Reproductive Health. Geneva: WHO. P. 90. 
Askew, I., Z. Matthews and R. Partridge. 2002. Going Beyond Research. A Key Issues Paper Raising Discussion Points Related to Dissemination, Utilization, and Impact of Reproductive and Sexual Health Research. New York: Population Council, FRONTIERS. 25 pp.

Behague, D., Tawiah, C., Rosato, M., Some, T., and Morrison, J. 2009. Evidence-based policymaking: the implications of globally-applicable research for context-specific problem-solving in developing countries. Soc Sci Med, 69(10), 1539-1546. doi: 10.1016/j.socscimed.2009.08.006

Bertrand, Jane T. and M. Celeste Marin. 2001. Operations Research: Measuring Its Impact on Service Delivery and Policy. FRONTIERS/Tulane University.

Bowen, S., and Zwi, A. B. 2005. Pathways to "evidence-informed" policy and practice: a framework for action. PLoS Medicine, 2(7), e166. doi: 10.1371/journal.pmed.0020166

Brambila, C., Ottolenghi, E., Marin, C., and Bertrand, J. T. 2007. Getting results used: evidence from reproductive health programmatic research in Guatemala. Health Policy and Planning, 22(4), 234-245. doi: 10.1093/heapol/czm013

Braveman, P. A., Egerter, S. A., Woolf, S. H., and Marks, J. S. 2011. When do we know enough to recommend action on the social determinants of health? American Journal of Preventive Medicine, 40(1 Suppl 1), S58-66. doi: 10.1016/j.amepre.2010.09.026

Brownson, R. C., Chriqui, J. F., and Stamatakis, K. A. 2009. Understanding evidence-based public health policy. American Journal of Public Health, 99(9), 1576-1583. doi: 10.2105/ajph.2008.156224

Burris, H., J. Parkhurst, Y. Adu-Sarkodie, and P. Mayaud. 2011. “Getting Research into Policy - Herpes Simplex Virus Type-2 (HSV-2) Treatment and HIV Infection: International Guidelines Fromulation and the Case of Ghana." Health Research Policy and Systems. 9(Suppl 1):55: 1-10.

Clar, C., Campbell, S., Davidson, L., and Graham, W. 2011. What are the effects of interventions to improve the uptake of evidence from health research into policy in low and middle-income countries? (Systematic review) (pp. 107pp): University of Aberdeen (UoA).

Cookson, R. 2005. Evidence-based policy making in health care: what it is and what it isn't. Journal of Health Services Research and Policy, 10(2), 118-121. doi: 10.1258/1355819053559083

Crewe, E. and J. Young. 2002. "Bridging Research and Policy: Context, Evidence and Links." ODI Working Paper 173. 24 pages.

Cross, H, N Jewell and Karen Hardee. 2001. "Reforming Operational Policies: A Pathway to Improving Reproductive Health Programs.” POLICY Occasional Paper. No. 7. Washington DC: The Futures Group International, POLICY Project.

Damschroder, LJ., DC Aron, RE Keith, SR Kirsh, JA Alexander, and JC Lowery. 2009. "Fostering Implementation of Health Services Research Findings into Practice: A Consolidated Framework for Advancing Implementation Science. Implementation Science. 4/1/50.

DFID. 2014. "What is the evidence on the impact of research on international development?: A DFID Literature Review.” UKAID Department for International Development.

Dobbins, M., D. Ciliska, R. Cockerill, J. Barnsley, and A. DiCenso. 2002. "A framework for the dissemination and utilization of research for health-care policy and practice." Online J Knowl Synth Nurs 9:7. 
Dopson, S. 2010. “Evidence-based Health Care and The Implementation Gap.” In Lyons, R.F. Ed. 2010. Using Evidence: Advances and Debates in Bridging Health Research and Action. Oxford: Atlantic Health Promotion Research Center. Pp. 72-80.

Eyben, Rosalind. 2013. Uncovering the Politics of 'Evidence' and 'Results': A Framing Paper for Development Practitioners. Prepared for the Politics of Evidence Conference in Brighton, U.K., April 2013.

Eyben, R., Guijt, I., Roche, C., Shutt, C., and Whitty, B. 2013. The Politics of Evidence Conference Report. Paper presented at the The Big Push Forward, UK. http://bigpushforward.net/wpcontent/uploads/2013/09/BPF-PoE-conference-report.pdf

FHI. 2008a. "Realizing the Potential of 'Champions' to Promote the Use of Research Findings." Case Studies in Research Utilization. Research Triangle Park, NC: FHI (now FHI 360).

FHI. 2008b. "Expanding the Community-based Distribution of Injectable Contraceptives in Africa." Case Studies in Research Utilization. Research Triangle Park, NC: FHI (now FHI 360).

FHI. 2008c. "Improving Contraceptive Access with a Simple Job Aid to Help Rule out Pregnancy.” Case Studies in Research Utilization. Research Triangle Park, NC: FHI (now FHI 360).

Fisher, A., J. Laing, J.E. Stoeckel, J.W. Townsend. 1991. Handbook, for Family Planning Operations Research Design. Second Edition. New York: Population Council.

Foreit, James R., and Tomas Frejka. 1998. Family Planning Operations Research: A Book of Readings. New York: The Population Council.

Fox, D. 2004. "Research to Policy: a Contrarian's View”. In WHO. 2004. World Report on Knowledge for Better Health: Strengthening Health Systems. Geneva: WHO. 146 pp.

Freedman, Ronald, and Bernard Berelson. 1976. "The record of family planning programs," Studies in Family Planning, 7(1): 1-40.

Freudenberg, n. and E. Tsui. 2014. "Evidence, Power, and Policy Change in Community-based Participatory Research.” American Journal of Public Health. 104(1): 11-14.

FRONTIERS in Reproductive Health. ND. Legacy Series: Capacity Building. Washington, DC: Population Council.

Glasgow, R.E. and K.M. Emmons. 2007. "How Can We Increase Translation of Research into Practice?? Types of Evidence Needed." Annual Review of Public Health. 28: 413-433.

Haaga, J.G. and R.M. Maru. 1996. "The Effect of Operations Research on Program Changes in Bangladesh.” Studies in Family Planning. 27(2): 76-87.

Haynes, A.S., G.E. Derrick, S. Redman, W.D. Hall, J.A. Gillespie, S. Chapman, and H. Sturk. 2012. "Identifying Trustworthy Experts: How Do Poliycmakers find and Assess Public Health Researchers Worth Consulting or Collaborating With?” PlosOne. 7(3): e32664, Pp. 1-8.

Healy, J., J. Maxwell, P.K. Hong, and V. Lin. 2007. Responding to Requests for Information on Health Systems from Policy Makers in Asian Countries. Geneva: Alliance for Health Policy and Systems Research.

Hillsman, Sally. 2015. “Communicating Social Science.” Footnotes. February 2015. 
Hoke, T., J. Haries, S. Crede, M. Green, D. Constant, T. Petruney, and J. Moodley. 2014. “Expanding Contraceptive Options for PMTCT Clients: A Mixed Methods Implementation Study in Cape Town, South Africa." Reproductive Health. 11:3_1-10.

Hunsmann, M. 2012. "Limits to Evidence-based Health Policymaking: Policy Hurdles to Structural HIV Prevention in Tanzania." Social Science \& Medicine. doi: 10.1016/j.sosscimed.2012.01.023.

Hyder, A.A., A. Corluka, P.J. Winch, A. El-Shinnawy, H. Ghassany, H. Malekafzali, M.K. Lim, J. MfutsoBengo, E. Segura and A. Ghaffar. 2010. "National Policymakers Speak Out: Are Researchers Giving Them What they Need?" Health Policy and Planning. 26: 73-82.

Innvaer, S., Vist, G., Trommald, M., \& Oxman, A. 2002. Health policymakers' perceptions of their use of evidence: a systematic review. Journal of Health Services Research and Policy, 7(4), 239-244. doi: $10.1258 / 135581902320432778$

Kay, J. 2010. Obliquity. London: Penguin Books.

Kerner, J. 2008. "Integrating Research, Practice, and Policy: What we See Depends on Where we Stand." Journal of Public Health Management Practice. 14(2): 193-198.

Kim, Julie. 2006. "WHO ProTest Initiative in South Africa: A Utilization Framework Case Study." Chapter in WHO. Department of Reproductive Health and Research. 2006. Turning Research into Practice: Suggested Actions from Case-studies of Sexual and Reproductive Health. Geneva: WHO. Pp. 51-56.

Koenig, M.A. and M. Whittaker. 1991. "Increasing the Application of Operations Research Findings in Public Sector Family Planning Programs: Lessons from the ICDDR,B Extension Project.” In Seidman, M. and M. Horn. 1991. Operations Research: Helping Family Planning Programs Work Better. Wiley Liss, Inc. Pp. 451-460.

Koon, A.D., K.D. Rao, N.T Tran and A. Ghaffar. 2013. "Embedding health policy and systems research into decision-making processes in low- and middle-income countries." Health Research Policy and Systems. 11/30: 1-9.

Kothari, A., and C.N. Wathen. 2013. "A Critical Second Look at Integrated Knowledge Translation.” Health Policy. 109: 187-191.

LaPelle, N.R. K. Dahlen, B. Gabella, A.L. Juhl, and E. Martin. 2014. “Overcoming Enertia: Increasing Public Health Departments' Access to Evidence-Based Information and Promoting Usage to Inform Practice." American Journal of Public Health. 104(1): 77-80.

Lavis, J. N., Rottingen, J. A., Bosch-Capblanch, X., Atun, R., El-Jardali, F., Gilson, L., et al. 2012. Guidance for evidence-informed policies about health systems: linking guidance development to policy development. PLoS Med, 9(3), e1001186. doi: 10.1371/journal.pmed.1001186

Law, S. 2010. "Collaboration for change—the long and winding road or, "No, honey, we're not there yet." In Lyons, R.F. Ed. 2010. Using Evidence: Advances and Debates in Bridging Health Research and Action. Oxford: Atlantic Health Promotion Research Center. Pp. 105-109.

Lewis, S. 2007. "Toward a General Theory of Indifference to Research-based Evidence." Journal of Health Services Research Policy. 12(3): 166-172. 
Liambila, W. et al. 2008. Feasibility, Acceptability, Effect and Cost of Integrating Counseling and Testing for HIV within Family Planning Services in Kenya. FRONTIERS Final Report, Washington, DC, Population Council.

Lomas, Jonathan. 1997. Improving Research Dissemination and Uptake in the Health Sector: Beyond the Sound of One Hand Clapping. McMaster University Centre for Health Economics and Policy Analysis Policy Commentary C97-1, November 1997.

Lomas, J., T. Culyer, C. McCutcheon, L. McAuley, and S. Law. 2005. Conceptualizing and Combining Evidence for Health System Guidance." Ottawa: Canadian Health Services Research Foundation.

McCoy, D., K. Storeng, V. Fillipi, C. Ronsmans, D. Osrin, B. Matthias, O.M. Campbell, R. Wolfe, A. Prost, Z. Hill, A, Costello, K. Azad, C. Mwansambo, and D.S. Manandhar. 2010. "Maternal, neonatal and child health interventions and services: moving from knowledge of what works to systems that deliver." International Health. 2: 87-98.

McKibbon, KA, C. Lokker, NL Wilczynski, D. Ciliska, M Dobbins, DA Davis, RB Haynes, and SE Straus. 2010. "A Cross-sectional Study of the Number and Frequency of Terms Used to Refer to Knowledge Translation in a Body of Health Literature in 2006: A Tower of Babel?” Implementation Science. 5(16). 11 pages.

MEASURE Evaluation. 2009. Making Research Findings Actionable. Chapel Hill, NC: MEASURE Evalutation.

Mijumbi, Rhona, Andrew Oxman, Ulysses Panisset, Nelson Sewankambo. 2014. Feasibility of a Rapid Response Mechanism to Meet Policymakers' Urgent Needs for Research Evidence About Health Systems in a Low Income Country: A Case Study. Implementation Science. 9:114. doi:10.1186/s13012-014-0114-z.

Moat, N., Lavis, J.N. 2012. 10 Best Resources for...evidence-informed health policy making. Health Policy and Planning, pp.1-4.

Moat, K.A., J.N. Lavis, S.J. Clancy, F. El-Jardali, and T. Pantoja. 2014. "Evidence Briefs and Deliberative Dialogues: Perceptions and Intentions to Act on What was Learnt." Bulletin of the World Health Organization. 4(92): 20-28.

Murthy L, Shepperd S, Clarke MJ, Garner SE, Lavis JN, Perrier L, Roberts NW, Straus SE. 2012. Interventions to improve the use of systematic reviews in decision-making by health system managers, policy makers and clinicians. Cochrane Database Syst Rev.

Nath, S. 2007. "Final Report: Getting Research into Policy and Practice (GRIPP).” London: JSI Europe and Washington, DC: Population Council FRONTIERS in Reproductive Health.

Neilson, S. 2001. Knowledge Utilization and Public Policy Processes: A Literature Review. Canada: Evaluation Unit, International Development Research Council.

Nutley, T. and H. Reynolds. 2013. "Improving the Use of Health Data for Health Systems Strengthening." Global Health Action. 6:20001. 10 pages.

Oliver, K., Innvar S., Lorenc T., Woodman J., and Thomas J. 2014. "A Systematic Review of Barriers to and Facilitators of the Use of Evidence by Policymakers." BMC Health Services Research. 14(2). 
Ongolo-Zogo, P., J.N. Lavis, G. Tomson, and N.K. Sewankambo. 2015. “Climate for Informed Health System Policymaking in Cameroon and Uganda Before and After the Introduction of Knowledge Translation Platforms: A Structured Review of Governmental Policy Documents." Health Research Policy and Systems. 13(2): 1-27.

Oxman, A. D., J. N. Lavis, S. Lewin, and A. Fretheim. 2009a. "SUPPORT Tools for evidence-informed health Policymaking (STP) 1: What is evidence-informed policymaking?" Health Res Policy Syst 7 Suppl 1:S1. doi: 10.1186/1478-4505-7-s1-s1.

Oxman, A. D., P. O. Vandvik, J. N. Lavis, A. Fretheim, and S. Lewin. 2009b. "SUPPORT Tools for evidence-informed health Policymaking (STP) 2: Improving how your organisation supports the use of research evidence to inform policymaking." Health Res Policy Syst 7 Suppl 1:S2. doi: 10.1186/1478-4505-7-s1-s2.

Oxman, A. D., J. N. Lavis, A. Fretheim, and S. Lewin. 2009c. "SUPPORT Tools for evidence-informed health Policymaking (STP) 17: Dealing with insufficient research evidence." Health Res Policy Syst 7 Suppl 1:S17. doi: 10.1186/1478-4505-7-s1-s17.

Paina, L., and Peters, DH. 2011. "Understanding Pathways for Scaling up Health Services Through the Lens of Complex Adaptive Systems.” Health Policy and Planning. Published online August 5. doi: 10.1093/heapol/czr054.

Panisset, U., Koehlmoos, T. P., Alkhatib, A. H., Pantoja, T., Singh, P., Kengey-Kayondo, J., and McCutchen, B. 2012. Implementation research evidence uptake and use for policymaking. Health Research and Policy Systems, 10, 20. doi: 10.1186/1478-4505-10-20

POLICY Project. 2005. Core Package Final Report: Determining the Feasibility and Potential Scope of Integrating Reproductive Health Services in Jamaica. Washington, DC: Futures Group, POLICY Project, 2005.

Rosenbaum, S. E., C. Glenton, C. S. Wiysonge, E. Abalos, L. Mignini, T. Young, F. Althabe, A. Ciapponi, S. G. Marti, Q. Meng, J. Wang, A. M. la Hoz Bradford, S. N. Kiwanuka, E. Rutebemberwa, G. W. Pariyo, S. Flottorp, and A. D. Oxman. 2011. "Evidence summaries tailored to health policymakers in low- and middle-income countries." Bull World Health Organ 89 (1):54-61. doi: 10.2471/blt.10.075481.

Ross, J. 1998. “Forward.” In Foreit, James R., and Tomas Frejka. 1998. Family Planning Operations Research: A Book of Readings. New York: The Population Council.

Seidman, Myrna, and Marjorie C. Horn. 1991. Operations Research: Helping Family Planning Programs Work Better. New York: Wiley-Liss, Inc.

Sheikh, K., A. George, and L. Gilson. 2014. "People-centered Science: Strengthening the Practice of Health Policy and Systems Research.” Health Research Policy and Systems. 12(19): 1-8.

Shelton, James D. 2014. Evidence-based Public Health: Not Only Whether it Works but How Can It Be Made to Work Practicably at Scale. Glob Health Sci Prac. 2014, 2(3):253-258. http://dx.doi.org/10.9745/GHSP-D-14-00066.

Simmons, R., Brown, J., and Diaz, M. 2002. Facilitating large-scale transitions to quality of care: an idea whose time has come. Studies in Family Planning, 33(1), 61-75. 
Simmons, R., Fajans, P., and Ghiron, L. 2007. Scaling up health service delivery: from pilot innovations to policies and programmes (pp. 205pp). Geneva: WHO.

Smith, Ellen, et al. 2015. Evidence for Family Planning Advocacy: An Assessment of Decisionmakers' and Advocates' Needs and Strategies in East Africa. Washington, DC: Futures Group, Health Policy Project.

Solo, J., N. Cerulli, R. Miller, I. Askew, and E. Pearlman. 1998. "Strengthening the Utilization of Family Planning Operations Research: Findings from Case Studies in Africa." Unpublished report from the Population Council FRONTIERS Project.

Spicer, N., D. Bhattacharya, R. Dimka, F. Fanta, L. Mangham-Jefferies, J. Schellenberg, A. TamirWoldemariam, G. Walt, and D. Wichremasinghe. 2014. "'Scaling-up is a Craft Not a Science': Catalysing Scale-up of Health Innovations in Ethiopia, India and Nigeria." Social Science and Medicine. 121: 30-38.

STEP UP Research Programme Consortium. 2013. Second Consultation on Developing Standards for Identifying Evidence -based Practices in Reproductive Health. STEP UP Report. New York: The Population Council. 17 pages.

Sumner, A., J. Crichton, S. Theobald, E. Zulu and J. Parkhurst. 2011. "What Shapes Research Impact on Policy? Understanding Research Uptake in Sexual and Reproductive Health Policy Processes in Resource Poor Contexts." Health Research Policy and Systems. 9(Suppl 1):53: 1-10.

Torfing, J. 2009. "Rethinking Path Dependence in Public Policy Research." Critical Policy Studies. 3(1): 70-83.

Trostle, J. 2006. "Pathways to promote and guide the use of sexual and reproductive health research." WHO. Department of Reproductive Health and Research. 2006. Turning Research into Practice: Suggested Actions from Case-studies of Sexual and Reproductive Health. Geneva: WHO. P. 44.

Trostle, J., M. Bronfman and A. Langer. 1999. "How Researchers Influence Decision-makers? Case Studies from Mexican Policies." Health Policy and Planning. 14(2): 103-114.

Ulin, P., E. Robinson and B. Tolley. 2005. Qualitative Methods in Public Health; A Field Guide for Applied Research. San Fransciso, C: Jossey-Bass.

University of Cambridge: Faculty of Education. 2014. Implementation Science. In Implementing Implementation Science: The science of making interventions effective in real world contexts. Cambridge: University of Cambridge. Retrieved from http://www.educ.cam.ac.uk/events/conferences/implementation/

USAID. n.d. “Discovery to Scale Up. Implementation Science in Global Health.” Washington, DC: USAID. Unpublished.

Wathen, C.N., J.C.D. MacGregor, S.L. Sibbald, and H.L. MacMillian. 2013. "Exploring the Uptake and Framing of Research Evidence on Universal Screening for Intimate Partner Violence Against Women: A Knowledge Translation Case Study." Health Research Policy and Practice. 11:13: 1-13.

WHO. 2004. World Report on Knowledge for Better Health: Strengthening Health Systems. Geneva: WHO. $146 \mathrm{pp}$. 
WHO. Department of Reproductive Health and Research. 2006. Turning Research into Practice: Suggested Actions from Case-studies of Sexual and Reproductive Health. Geneva: WHO.

Yamey, G., and Feachem, R. 2011. Evidence-based policymaking in global health - the payoffs and pitfalls. Evidence Based Medicine, 16(4), 97-99. doi: 10.1136/ebm.2011.100060 


\section{The Evidence Project}

\section{Population Council}

4301 Connecticut Avenue, NW, Suite 280

Washington, DC 20008 USA

tel +12022379400

evidenceproject.popcouncil.org 